\title{
CORRESPONDENCE Identification of druggable inhibitory immune checkpoints on Natural Killer cells in COVID-19
}

\author{
Olivier Demaria ${ }^{1}$, Julien Carvelli ${ }^{2}$, Luciana Batista ${ }^{1}$, Marie-Laure Thibult ${ }^{1}$, Ariane Morel ${ }^{1}$, Pascale André ${ }^{1}$, Yannis Morel ${ }^{1}$, \\ Frederic Vély ${ }^{3,4}$ and Eric Vivier (iD) $1,3,4$
}

Cellular \& Molecular Immunology (2020) 17:995-997; https://doi.org/10.1038/s41423-020-0493-9

Infection with SARS-COV-2 is the cause of COVID-19 and has generated an unprecedented health crisis worldwide. While most of the patients experience mild symptoms, around 20\% develop severe disease, characterized by pneumonia and in the worst cases by acute respiratory distress syndrome (ARDS). ${ }^{1}$ The analysis and understanding of the immune responses arising in the course of SARS-COV-2 infection may help to propose therapeutic solutions. Due to the crucial role of Natural Killer (NK) cells in antiviral immune responses, ${ }^{2}$ we analyzed NK cells in blood from a cohort of 82 individuals: 10 healthy controls (HC), 10 paucisymptomatic COVID-19 patients (pauci), 34 patients with pneumonia (pneumo) and 28 patients with ARDS due to SARS-CoV-2 infection. The absolute numbers of peripheral blood NK cells, $\mathrm{B}, \mathrm{CD} 4^{+}$, and $\mathrm{CD}^{+} \mathrm{T}$ lymphocytes were lower in the pneumonia and ARDS groups than in healthy controls, consistent with previously published results ${ }^{3}$ (Fig. 1a). We investigated the NK cell subsets further and found that among $\mathrm{CD}_{4} 5^{+} \mathrm{CD} 3^{-} \mathrm{CD} 56^{+}$total NK cells the proportion of mature NK cells, a subset defined on the basis of its expression of the CD16 and CD57 cell surface receptors, was markedly lower in patients with ARDS (Fig. 1b). Given their role in viral infection, the loss of mature NK cells may contribute to the pulmonary complications occurring in the most severe cases of COVID-19. We then focused our analysis on molecular pathways likely to improve NK cell antiviral activity to promote SARS-CoV-2 clearance, and analyzed the expression of several immune checkpoints. Given the availability of therapeutic monoclonal antibodies blocking the immunosuppressive functions of PD-1, ${ }^{4}$ NKG $2 A^{5}$ and CD $39^{6}$ initially developed for cancer therapies, we analyzed the expression of these molecules on NK cells in our cohort. PD-1 and NKG2A are cell surface receptors, and their engagement with their ligands, PD-L1 and HLA-E, respectively, inhibits the function of T and NK cells. CD39 is an ectoenzyme that cleaves extracellular ATP and ADP, which can be released from dead cells upon viral infection, leading to the generation of adenosine, which has strong immunosuppressive effects on $\mathrm{T}$ and NK cells.

We observed the presence of a CD39-expressing NK cell population in the blood of the COVID-19 patients of the pneumonia and ARDS groups that was absent in the HC and paucisymptomatic groups. Moreover, the cell surface density of CD39, which is known to be upregulated by hypoxia, was much higher in this subset than observed in normal conditions, as shown by the small number of CD39-expressing NK cells from HC (Fig. 1C). IL-6 induces CD39 expression on tumor infiltrating NK cells and might participate to their impaired functions in the context of cancer. ${ }^{7}$ Expression of CD39 on NK cells from COVID19 patients may thus be explained by the levels of circulating IL6 that rise with disease severity. Similarly, the PD-1 receptor was upregulated on NK lymphocytes in COVID-19 patients, and several pneumonia and ARDS COVID-19 patients had a particularly large subset of NK cells expressing PD-1. The expression of PD-1 on NK cells has been described in some human cancers, but a high frequency of PD-1 ${ }^{+}$NK cells, as observed in the peripheral blood of some severely affected COVID-19 patients, is uncommon. We found that the NKG2Aexpressing NK cell subset was smaller in patients with ARDS, although the cell surface density of NKG2A was upregulated in this group (Fig. 1C). By contrast, the expression of NKG2C, an activating receptor of HLA-E implicated in the control of CMV infections, remained unmodified in COVID-19 patients (Fig. 1C). Among the inhibitory or activating receptors for HLA-C, the expression of KIR2DL1/S1 was also stronger on the NK cells of ARDS patients, whereas KIR2DL2/L3/S2 levels remained unmodified (Fig. 1C). Thus, circulating NK cells in COVID-19 patients display an upregulation of the inhibitory receptor NKG2A that is associated with disease severity, consistent with recent results. ${ }^{8}$ Bronchoalveolar lavage fluid (BALF) analysis showed a lack of $\mathrm{CD} 16^{+} \mathrm{CD} 57^{+}$mature NK cells in the lungs of ARDS patients (Fig. 1d), suggesting that the decrease in mature NK cell levels observed in blood (Fig. 1b, d) is not a consequence of their migration to infected lungs. In addition, high levels of CD39, PD1 , and NKG2A expression were also observed in NK cells isolated from the BALF of ARDS COVID-19 patients, and these levels were even higher than those observed on blood cells of the patients tested (Fig. 1e, f). Together, these results show that NK cells in the blood and BALF of COVID-19 patients display signs of dysfunction resembling those described in cancers, at least to some extent. The blockade of CD39, PD-1, or NKG2A has been shown to harness NK cell immunity in cancers and would be of interest in COVID-19, to enhance NK cell-mediated viral clearance. We therefore analyzed whether NK cells from COVID-19 patients remained functional and could be reinvigorated. We found that NK cells isolated from the blood of ARDS COVID-19 patients retained cytotoxic functions, and that

\footnotetext{
${ }^{1}$ Innate Pharma, 117 avenue de Luminy, Marseille 13009, France; ${ }^{2}$ Assistance Publique des Hôpitaux de Marseille, Hôpital de la Timone, Réanimation des Urgences, Marseille 13005, France; ${ }^{3}$ Aix Marseille Université, CNRS, INSERM, Centre d'Immunologie de Marseille-Luminy, Marseille 13009, France and ${ }^{4}$ Assistance Publique des Hôpitaux de Marseille, Hôpital de la Timone, Immunology, Marseille Immunopole, Marseille 13005, France

Correspondence: Olivier Demaria (olivier.demaria@innate-pharma.fr)

These authors contributed equally: Olivier Demaria, Julien Carvelli
}

Received: 15 June 2020 Accepted: 18 June 2020

Published online: 1 July 2020 


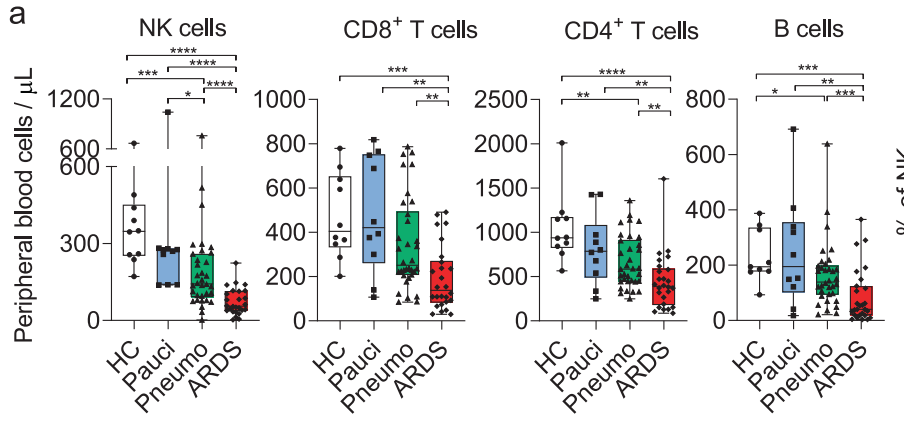

b

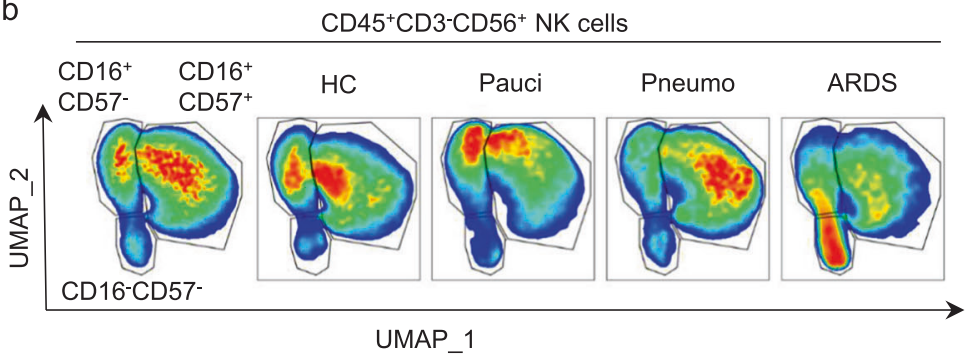

C
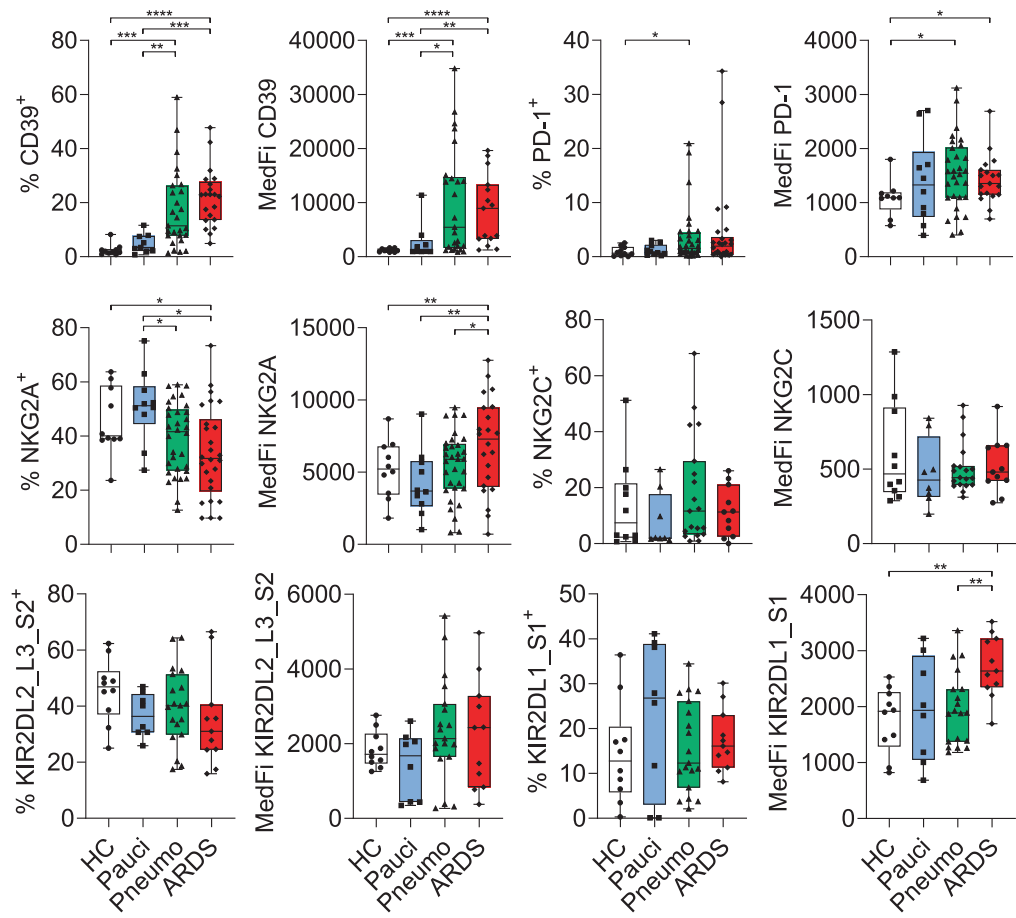

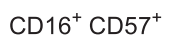

CD $16^{+}$CD57

$\mathrm{CD}^{-} 6^{-} \mathrm{CD} 57^{-}$
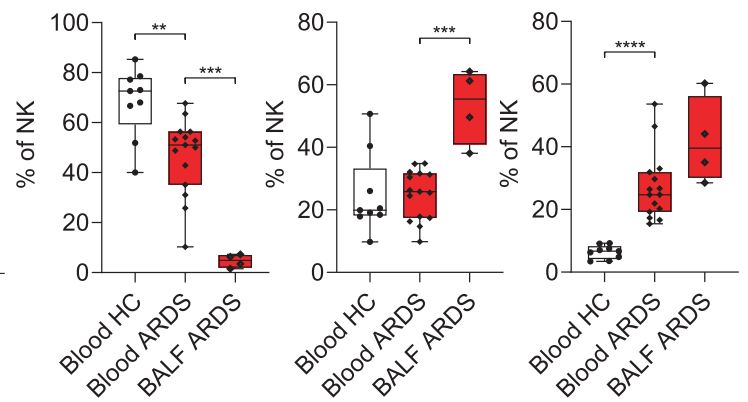

e

BALF NK cells

f

NK cells
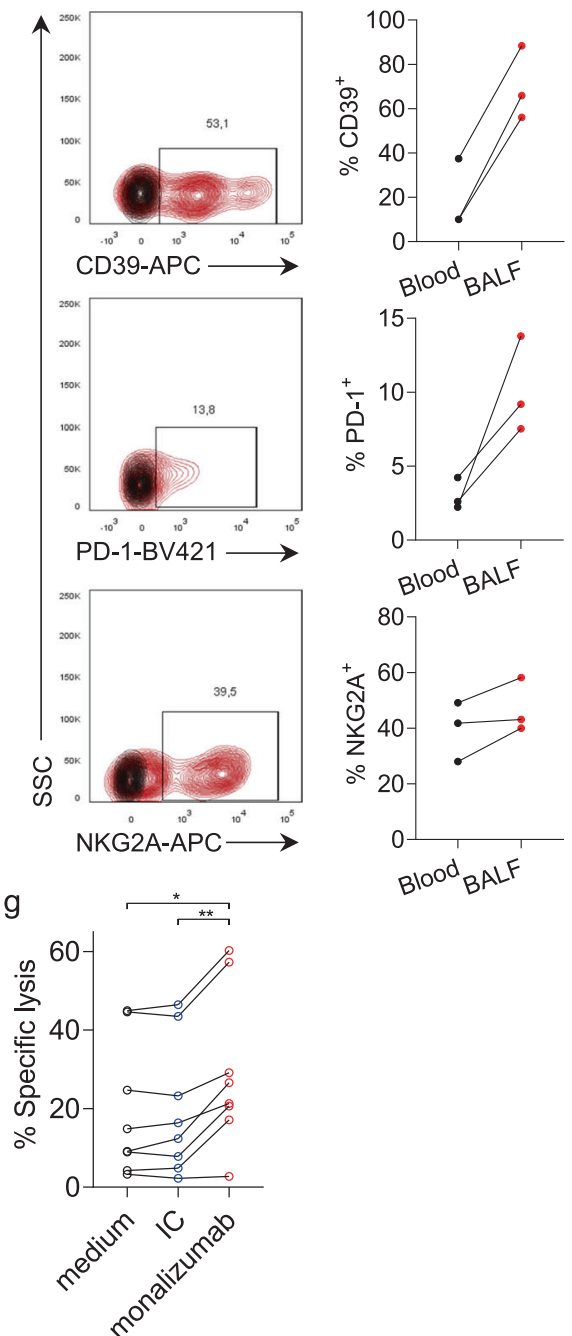

Fig. 1 COVID-19 is associated with lymphopenia and a dysfunctional NK cell phenotype. a Absolute numbers of circulating NK, B, CD4 ${ }^{+}$and $\mathrm{CD}^{+} \mathrm{T}$ cells per microliter of peripheral blood from healthy donors and COVID-19 patients. b Left: UMAP projection of concatenated

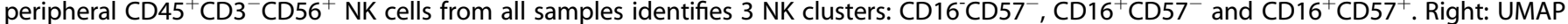
projections of concatenated peripheral blood NK cells from healthy donors and COVID-19 patients. Red indicates the highest cell density. c Phenotyping of NK cells in peripheral blood from healthy donors and COVID-19 patients. The percentages of total NK cells and MedFI for marker-positive NK cells are shown. $d$ Percentages of $\mathrm{CD} 16^{+} \mathrm{CD} 57^{+}, \mathrm{CD} 16^{+} \mathrm{CD} 57^{-}$, and $\mathrm{CD} 16^{-} \mathrm{CD}^{-} 7^{-}$subsets in $\mathrm{CD} 45^{+} \mathrm{CD} 3^{-} \mathrm{CD} 56^{+} \mathrm{NK}$ cells in the blood of HCs (white) and ARDS patients (red) and BALF from ARDS patients (red). e Representative flow cytometry contour plots from three ARDS patients showing CD39, PD-1, and NKG2A expression (red) vs. fluorescence minus one (FMO, black) on NK cells from BALF. f Percentages of CD39+ ${ }^{+}$PD $-1^{+}$and NKG2A ${ }^{+}$NK cells, cells in blood (black) and BALF (red) from the same ARDS patients. g Specific K562-HLA-E lysis by NK cells isolated from ARDS PBMCs incubated with medium (black), isotype control (IC, blue) or monalizumab (red). a-g, Each symbol represents a single donor. Experimental conditions and statistics details are provided in supplementary material. 
incubation with monalizumab, ${ }^{5}$ an anti-NKG2A mAb blocking the inhibitory interaction with $\mathrm{HLA}-\mathrm{E}$, was able to unleash their killing ability (Fig. 1g). These data suggest that therapeutic interventions may improve NK cell functions, facilitating virus elimination, and support the repurposing of therapeutic mAbs targeting inhibitory molecules in COVID-19. The immune system plays an important role in virus elimination in COVID-19, but it is becoming clear that the infiltration and activation of myeloid cells in infected lungs are also critical for ARDS development. ${ }^{9}$ Highly activated NK cells have been shown to worsen lung injury in respiratory infection models, and the use of therapeutic tools to improve NK cell functions should, thus, be carefully considered in severe cases of COVID-19. ${ }^{10}$ However, our data, showing a decrease in NK cell numbers and a dysfunctional state of these cells in the blood and lungs of COVID-19 patients, suggest that NK cells do not participate in the exaggerated inflammatory response observed in ARDS. Thus, therapies targeting PD-(L)1, NKG2A and CD39 should be investigated as means of boosting NK cell antiviral immunity in patients at early stages of SARS-CoV-2 infection.

\section{ACKNOWLEDGEMENTS}

We thank all the healthcare workers involved in the analysis, diagnosis and treatment of patients at AP-HM and Hôpital Laveran. We thank the Explore COVID-19 IPH group and the Explore COVID-19 Marseille-Immunopole group described in the supplementary material. The E.V. laboratory at CIML and Assistance-Publique des Hôpitaux de Marseille is supported by funding from the European Research Council (ERC) under the European Union's Horizon 2020 research and innovation program (TILC, grant agreement No. 694502 and MInfla-TILC, grant agreement No. 875102-MInflaTilc), the Agence Nationale de la Recherche including the PIONEER Project (ANR-17RHUS-0007), MSDAvenir, Innate Pharma and institutional grants to the CIML (INSERM, CNRS, and Aix-Marseille University) and Marseille Immunopole.

\section{AUTHOR CONTRIBUTIONS}

J.C., F.V. and E.V. initiated and designed the research. O.D. and E.V. wrote the manuscript with the help of the other coauthors. O.D., L.B., M-L.T., A.M., P.A., Y. M., and F.V. performed the experiments and analyzed and/or interpreted results. J.C. was in charge of patient care and contributed to the discussion of the results.

\section{ADDITIONAL INFORMATION}

The online version of this article (https://doi.org/10.1038/s41423-020-0493-9) contains supplementary material.
Competing interests: O.D., L.B., M.-L.T., A.M., P.A., Y.M., and E.V. are employees of Innate Pharma. None of the other authors has any competing financial interests to declare.

\section{REFERENCES}

1. Zhu, N. et al. A novel coronavirus from patients with pneumonia in China, 2019. N. Engl. J. Med. 382, 727-733 (2020).

2. Hammer, Q., Ruckert, T. \& Romagnani, C. Natural killer cell specificity for viral infections. Nat. Immunol. 19, 800-808 (2018).

3. Wang, F. et al. Characteristics of peripheral lymphocyte subset alteration in COVID-19 pneumonia. J. infect. dis. https://doi.org/10.1093/infdis/jiaa150 (2020).

4. Xin, Yu,J. et al. Trends in clinical development for PD-1/PD-L1 inhibitors. Nat. Rev. Drug Discov. 19, 163-164 (2020).

5. Andre, $\mathrm{P}$. et al. Anti-NKG2A mAb Is a checkpoint inhibitor that promotes antitumor immunity by unleashing both T and NK cells. Cell 175, 1731-1743 e1713 (2018).

6. Perrot, l. et al. Blocking antibodies targeting the CD39/CD73 immunosuppressive pathway unleash immune responses in combination cancer therapies. Cell Rep. 27, 2411-2425 e2419 (2019).

7. Zheng, Y. et al. IL-6-induced CD39 expression on tumor-infiltrating NK cells predicts poor prognosis in esophageal squamous cell carcinoma. Cancer Immunol. Immunother. https://doi.org/10.1007/s00262-020-02629-1 (2020).

8. Zheng, M. et al. Functional exhaustion of antiviral lymphocytes in COVID-19 patients. Cell. Mol. Immunol. https://doi.org/10.1038/s41423-020-0402-2 (2020).

9. Merad, M. \& Martin, J. C. Pathological inflammation in patients with COVID-19: a key role for monocytes and macrophages. Nat. Rev. immunol. https://doi.org/ 10.1038/s41577-020-0331-4 (2020).

10. Rajaram, S. et al. The promise and peril of natural killer cell therapies in pulmonary infection. Immunity. https://doi.org/10.1016/j.immuni.2020.04.018 (2020).

(i) Open Access This article is licensed under a Creative Commons Attribution 4.0 International License, which permits use, sharing, adaptation, distribution and reproduction in any medium or format, as long as you give appropriate credit to the original author(s) and the source, provide a link to the Creative Commons license, and indicate if changes were made. The images or other third party material in this article are included in the article's Creative Commons license, unless indicated otherwise in a credit line to the material. If material is not included in the article's Creative Commons license and your intended use is not permitted by statutory regulation or exceeds the permitted use, you will need to obtain permission directly from the copyright holder. To view a copy of this license, visit http://creativecommons. org/licenses/by/4.0/.

(c) The Author(s) 2020 